\title{
MedienPädagogik
}

Zeitschrift für Theorie und Praxis der Medienbildung

Jahrbuch Medienpädagogik 3.

Zweitveröffentlichung aus: Jahrbuch Medienpädagogik 3. (2003) Opladen: Leske + Budrich. Herausgegeben von Ben Bachmair, Peter Diepold und Claudia de Witt.

\section{Visuelle Argumentation: Schlüsselbilder im Selbstverständnis von Kulturen}

\author{
Manfred Behr
}

\section{Schlüsselbilder: Bilder im Kopf}

Beim Intermedia-Kongress 1985 in Hamburg fand eine Bilderausstellung statt, die kein einziges Bild zeigte: „Bilder im Kopf. Pictures in our Minds“. Auf schwarzen Tafeln konnten die Besucher die Beschreibungen von 40 Fotos lesen, zum Beispiel „Albert Einstein streckt die Zunge heraus“, „Nacktes Vietnamesen-Kind, das nach einem Napalm-Angriff aus dem Dorf Trang Bang flüchtet“, „Willy Brandt knieend am Ehrenmal der Helden des Warschauer Ghettos“. Man mag nicht einmal die Einzelheiten kennen (welches Ehrenmal?) - die Bilder entstehen im Kopf des Betrachters. „Die Fotos gingen um die Welt. Sie haben uns erschreckt, aufgerüttelt, belustigt. Die Bilder haben eines gemeinsam: Sie entrinnen dem Kopf des Betrachters und der Betrachterin so schnell nicht wieder“, so der damalige Erste Bürgermeister Klaus von Dohnanyi. Es sind Schlüsselbilder, Bestandteile des imaginären Museums, sie sind nicht in den Köpfen der einzelnen Leute, „sondern im kollektiven, gemeinsamen Gedächtnis gespeichert. Mein individuelles Wiedererkennen ist ein Abrufen des kollektiven, hier visuellen Gedächtnisses“ (Kutter 1987, S. 104). Archetypen der Moderne? Sie sind „im Kopf, nicht in den Köpfen“ (Kutter 1987, S. 104), sagen „etwas Wesentliches über unsere Lage“ (Kutter 1987, S. 106) - unbestritten, aber wie tun sie das und warum so erfolgreich?

Ebenfalls in Hamburg proklamierte Aby Warburg die „Menschenrechte des Auges“; wenn seit geraumer Zeit die „Visuelle Zeitenwende“, der „pictoral turn“ ausgerufen wird, sollte dazu gehören, dass ich begreife, was mich ergreift, der klassische Leitsatz der Hermeneutik, nun aber bezogen auf Bilder. Wenn Schlüsselbilder im obigen Verständnis Argumentationskerne konstituieren, so ist damit im folgenden nicht das durative, das sich geringfügig variierende Bild als visuelle Habitualisierung (händeschüttelnde Politiker etc.) gemeint. Diese Auffassung lässt sich ebenfalls mit guten Gründen vertreten (Ludes 2001); sie ist der hier vorgestellten Auffassung komplementär.

Wenn „Pictures in our Minds“ in die unmittelbare Gegenwart verlängert würde, so wäre eine eigene Schauwand dem 11. September zu widmen. Kein punktuelles Ereignis in unserer aller Lebenszeit hat die Aufmerksamkeit der 
Weltöffentlichkeit so simultan erreicht, ja Weltöffentlichkeit im genauen Wortsinne überhaupt erst hergestellt, wie die Terroranschläge auf das World Trade Center. Gewiss unterstellt Florian Rötzer zu recht, dass der Anschlag gerade um dieser medialen Omnipräsenz willen von den Attentätern so minutiös inszeniert und „getimt“ wurde (Rötzer 2001). Aber aus den abertausenden Bildern von Augenzeugen, die aufgenommen und ausgestellt wurden (z.B. www.hereisnewyork.org), qualifizieren sich nur wenige zu Schlüsselbildern, deren Platz im imaginären Museum - jetzt darf man wohl ohne Pathos sagen - der Menschheit` sicher ist. Ich untersuche eines davon, nicht das sich nähernde zweite Flugzeug, nicht die Explosion, die Staubfrau, den Einsturz, ... - alle diese kämen auch in Frage und hätten die vergleichbare Struktur einer immanenten Dialektik. Als äußert subtil und anschlussfähig für Folgediskurse erwies sich die hier untersuchte Fotografie der Ruine von Frank Schwere (Agentur Matrix, Berlin), Titelbild unter anderem des SPIEGEL (Nr. 43/22.10.01) und der GEO-EPOCHE (Nr. 7/2001). An diesem registrierenden Abbild und gestalteten Bild lässt sich die Qualifikation von Schlüsselbildern des kollektiven Gedächtnisses synchron (strukturell) und diachron (historisch) als eine bildhafte Argumentation entfalten, die Deutungsmuster nahelegt, Bewusstsein prägt, Weltbilder subtil befestigt, gerade weil Bilder am sichersten dann erinnert werden, wenn sie im Gedächtnis als Bedeutung gespeichert wurden (Anderson 2001, S. 144).

Das Verständnis visueller Argumentationen liefert einen Beitrag zur systematischikonologischen und historisch-ikonografischen Fundierung einer Bildwissenschaft im Sinne politischer Ikonografie. Als solche sollte sie den Beitrag von Bilderwelten zu Bildern der Welt kritisch hinterfragen, die, wenn nicht gleich Kampf, so doch Missverständnisse, Feindbilder zwischen Kulturen befördern und Verständigung im - verbal geführten - interkulturellen Dialog behindern. Bildsprache beeinflusst vorbewusst (präattentiv), unabhängig davon, ob man des Lesens und Schreibens der Schriftsprache mächtig ist oder nicht. Ihre Suggestion ist der reflektierten Analyse vorgeschaltet, wir beherrschen sie passiv, durchschauen ihr Funktionieren aber in aller Regel nicht aktiv ganz gleich, ob das Bild als Plakat einer Demonstration vorangetragen, als Aufdruck auf einem T-Shirt wütend verbrannt wird oder einem anspruchsvollen Zeitschriftenartikel als Blickfang voransteht.

Schlüsselbilder, so die Ausgangsthese, funktionieren als implizite Argumente. Das Paradox liegt in der Lesbarkeit ihrer Sprachlichkeit, die sich gleichwohl gegen rationale Durchdringung immunisiert. Zur Offenlegung dieser Sprachlichkeit sind eingangs drei Begriffe aus der Sprachwissenschaft, die nicht als bekannt vorausgesetzt werden können, auf Bilder zu übertragen und auf ihre Tauglichkeit am oben genannten Beispiel zu überprüfen. 


\section{Terminologische Vorklärung: Proposition; Argumentation; Extension und Intension als Bedeutungsdimensionen}

Wenn man verbale und visuelle Kommunikation unter dem gemeinsamen Dach des Kommunizierens untersucht, so erweisen sich drei Termini als effizient:

- die Proposition als Grundstruktur von Argumenten

- die Argumentationstypen des deduktiven und des induktiven Argumentes

- Extension und Intension als Dimensionen der Bedeutung.

\section{Propositon}

Wenn wir einen Inhalt denken oder sprachlich ausdrücken, müssen wir stets zwei Arten von Handlungen vollziehen: Zum einen müssen wir auf ein Objekt oder auf mehrere Objekte Bezug nehmen -, immer müssen wir über etwas nachdenken oder sprechen. Zum anderen müssen wir über die Objekte, auf die wir Bezug nehmen, etwas aussagen; wir müssen zum Ausdruck bringen, dass sie bestimmte Eigenschaften haben oder in bestimmten Beziehungen zueinander stehen.

Man nennt die Bezugnahme auf Objekte Referenz -, das Ausdrücken vön Eigenschaften einzelner Objekte oder von Beziehungen zwischen Objekten nennt man Prädikation. Eine vollständige Aussage, die sowohl referierende als auch prädizierende Teilausdrücke enthält, nennt man auch Proposition. (Bayer 1999, S. 186). Mit der einfachsten Formulierung: Die Proposition besteht darin, etwas über etwas auszusagen bzw.: Von Konzept A wird Konzept B ausgesagt. Propositionen bilden die morphologischen Bausteine von Argumentationen, die kleinsten Bestandteile, über deren Zutreffen oder Nicht-Zutreffen sinnvoll reflektiert werden kann.

\section{Argumentation}

„Argumentation“ so Jürgen Habermas „,nennen wir den Typus einer Rede, in dem die Teilnehmer strittige Geltungsansprüche thematisieren und versuchen, diese mit Argumenten einzulösen oder zu kritisieren. Ein Argument enthält Gründe, die in systematischer Weise mit dem Geltungsanspruch einer problematischen Äußerung verknüpft sind. Die ,Stärke eines Arguments bemisst sich, in einem gegebenen Kontext, an der Triftigkeit der Gründe; diese zeigt sich u.a. daran, ob ein Argument die Teilnehmer eines Diskurses überzeugen, das heißt zur Annahme des jeweiligen Geltungsanspruchs motivieren kann“ (Habermas 1985, S. 38).

Habermas' Vertrauen auf den zwanglosen Zwang des besseren Argumentes zielt auf die verbale diskursive Verständigung in der face-to-face-Kommunikation. Bei Bildern treten die Zeiträume und Orte des Entstehens 
und der Betrachtung auseinander; nur in speziellen Situationen wird etwa die veranschaulichende Skizze zum Zwecke besserer Verständlichkeit eingesetzt. Zwei Argumentationstypen sind zu unterscheiden: das deduktive und das induktive Argument.

Deduktiv verfahren Argumente nach dem Muster:

Jedes Säugetier hat ein Herz.

Alle Pferde sind Säugetiere.

Jedes Pferd hat ein Herz.

Induktiv: Jedes der Pferde, die bisher beobachtet worden sind, hat ein Herz gehabt.

Jedes Pferd hat ein Herz (Salmon 2001, S. 33).

Jedes Argument besteht aus einer Konklusion und einer oder meist mehreren Prämissen. Die Konklusion ist eine Aussage, die durch die Prämissen gestützt werden soll. Die Prämissen sind Aussagen, die - bei Anfechtung - als Gründe zur Stützung der Konklusion angegeben werden (Bayer 1999, S. 87). Da in den Prämissen Sachverhalte behauptet werden, können Prämissen wahr oder falsch sein. Sind sie falsch, so handelt es sich nur um angebliche Gründe; sie können deshalb die Konklusion nicht stützen. Das Urteil über die Wahrheit oder Falschheit der Prämissen als Bezug zu außersprachlicher Wirklichkeit ist allerdings keine Aufgabe der Logik. Bei deduktiven Argumenten ist die Konklusion in den Prämissen vollständig enthalten, deduktive Argumente bilden daher im Grunde nur Ausformulierungen der Teilmenge eines größeren Ganzen.

Interessanter sind induktive Argumente. Sie rechnen etwas hoch oder setzen etwas fort, dessen Verallgemeinerung oder Übertragung möglicherweise nicht statthaft ist. Sie besitzen Konklusionen, deren Gehalt über den Gehalt ihrer Prämissen mehr oder weniger weit hinausgeht (Salmon 2001, S. 163) Bildhafte induktive Argumente suggerieren eine Bedeutungsübertragung, ohne diese in ihrer Logik transparent zu machen. Sie sind daher scheinbar zwingend, bei näherer Analyse enthüllt sich aber in aller Regel ihre Suggestivität als angreifbar. Induktive Argumente sind notwendig, um Wissen zu erweitern. Sie bahnen Pfade in das Ungesicherte. Da der Gehalt Ihrer Konklusionen den Gehalt Ihrer Prämissen übersteigt, können Sie den Notwendigkeitscharakter deduktiver Argumente nicht besitzen. Im Gegensatz zu einem deduktiven Argument kann ein logisch korrektes induktives Argument von wahren Prämissen ausgehen, aber zu falschen Konklusionen führen. Die durch Extrapolation oder Transfer begründeten Schlussfolgerungen behalten folglich stets einen Rest an Hypothetischem.

Extension und Intension als Bedeutungsdimensionen

Begriffe können nach zwei Aspekten betrachtet werden: Nach ihren Inhalt und nach ihrem Umfang. Synonym für Inhalt und Umfang von Begriffen sind die Bezeichnungen Intension und Extension. „Die Intension eines Begriffes 
ist dabei der Begriffsinhalt, die Extension aber die Klasse der Dinge, die unter diesen Begriff fällt. (...) Diese Erklärung kann folgendermaßen veranschaulicht werden: Der Begriff ,Student' kann durch eine Anzahl von Merkmalen wie ,an einer Hochschule immatrikuliert, in einem wissenschaftlichen Lernprozess stehend, etc.' expliziert werden. Diese Merkmale müssen so beschaffen sein, dass sie genau die essentiellen Bedingungen - die unbedingt zur Etablierung dieses Begriffs Student gehören - erfüllen. Sie sind von unwesentlichen Merkmalen, die akzidentiell zur Charakterisierung einzelner Studenten hinzu kommen können (männlich, weiblich, Alter, Kleidung, etc...) scharf zu trennen. Die Extension des Begriffs ,Student` dagegen, kann einfach als die Klasse oder die Menge aller Studenten aufgefasst werden. Dabei wird die Abhängigkeit des Begriffs ,Extension` von den Begriff ,Intension deutlich: Wir können nur dann von der Klasse der Studenten sprechen, wenn wir wissen, welche essentiellen Merkmale, also welcher Begriffsinhalt zur Charakterisierung eines Studenten gegeben ist“ (Brekle 1972, S. 56f.).

Mit der analytischen Trennung der Bedeutungsdimensionen lassen sich viele der epistemologischen Probleme ausräumen, die jetzt noch die Diskussion um die Bildsemantik verunklären.

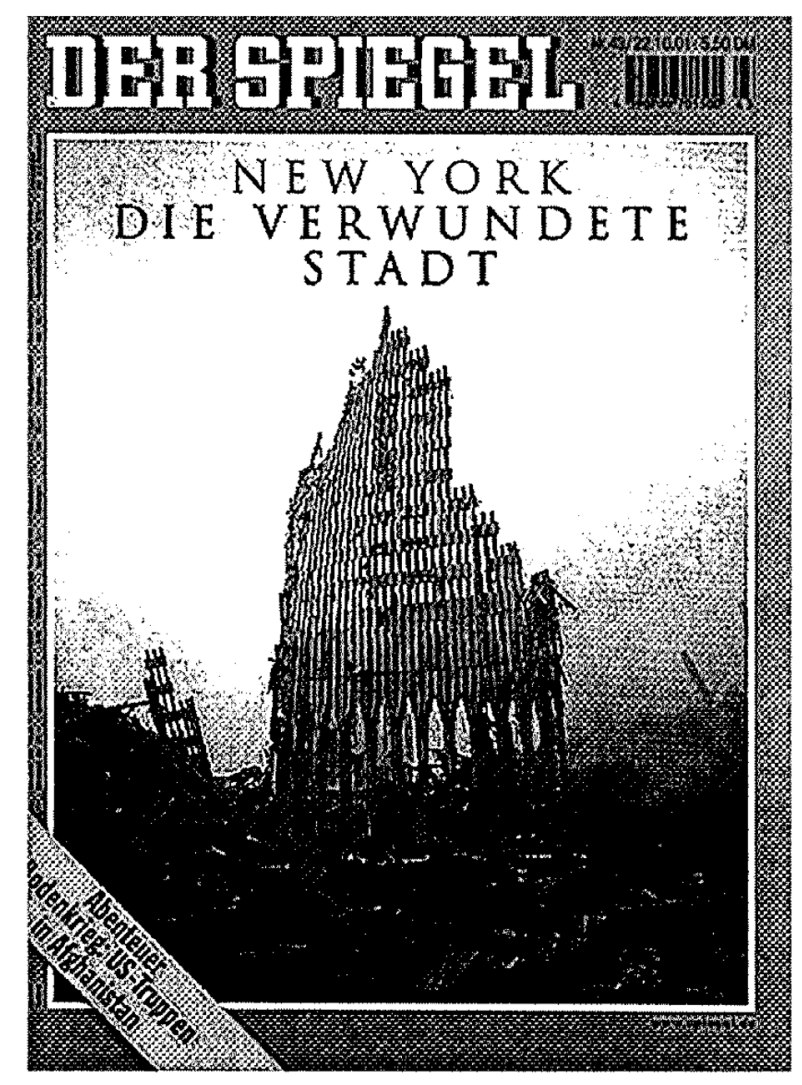




\section{Bedeutungsverdichtung im Schlüsselbild}

Mit Hilfe der vorstehend rekapitulierten Begriffe lässt sich das Titelbild des SPIEGEL semiotisch erschließen. Noch nichts ist damit bewiesen über sein Potential als Schlüsselbild - dies wird in einer zweiten, der metaphorischen Analyse zu untersuchen sein.

Erhellend ist sicherlich der Vergleich mit einer der unzähligen, hermeneutisch schwächeren Fotografien der Ruine. Auf dem nachstehenden Bildausschnitt wirkt die Ruine beliebig; wir werden informiert, spüren aber nicht die eigenständige, bedeutungssteigernde Kraft der bildnerischen Mittel, der visuellen Inszenierung, die das referierende Abbild semantisch zum über sich hinausweisenden Bild aufwertet.

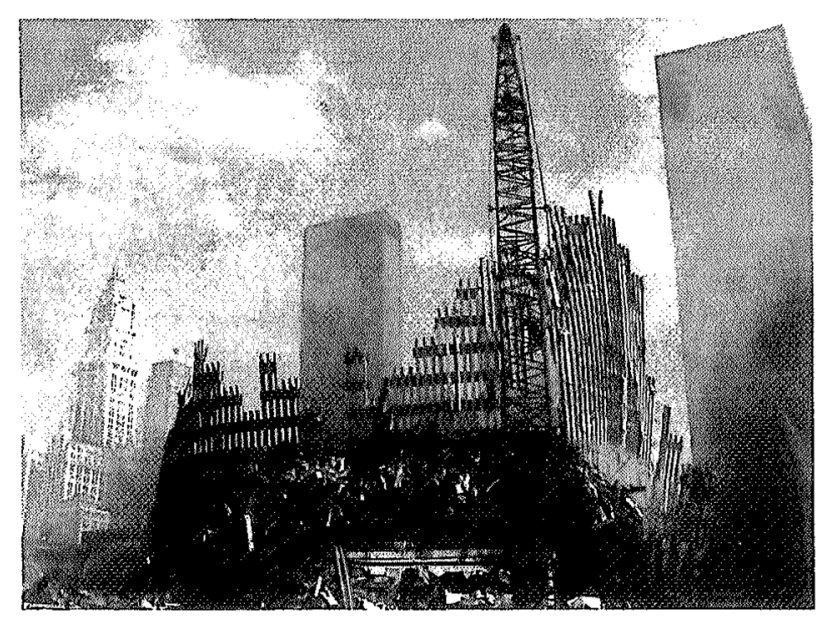

Gerade das von Frank Schwere gewählte Trümmerstück muss von besonderem Ausdruck und gesteigerter Ausstrahlung sein, anders wäre nicht zu erklären, warum genau dieses in dieser Gestalt auf dem ground zero als Mahnmal wieder errichtet werden soll (Chronik aktuell. Der 11. September 2001, S. 46).

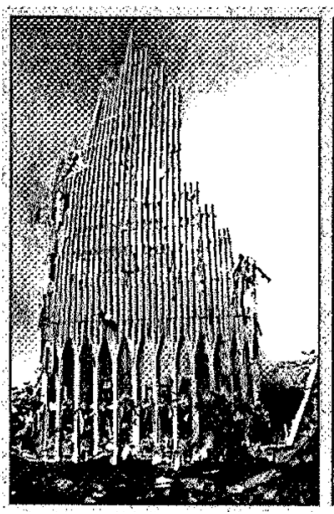

\section{WTC-Mahnmal}

In stundenlanger Nachtarbeit haben Bergungstrupps mit Schwerlastkränen das sieben stockwerke hohe Reststúck des World Trade Centers eborgen. Die Metallfassade, die inmer wieder fotografiert und im Fernsehen gezeigt wrurde, soll spáter als Mahnmal gegen den Terrorismus aurgestellt werden mus aur fllungen der Norker Stadrerwaltung kann das Fassadenstiuck noglicherweise auch in Rahmen der Neubebauung des WTC-Grund stickes deutlich sichtbar installiert werden. 
Gerade dieser Ruinenteil scheint in der Betrachtung die geistige Verbindung, das „missing link“ zwischen dem Gewesenen des Bauwerks und seiner imaginativen Vergegenwärtigung in der Erinnerung des Betrachters leisten zu können. Anders wäre der Ansporn zur Wiedererrichtung einer (im Gegensatz zum noch stehenden Rumpf der Berliner Gedächtniskirche) bereits sorgfältig zerlegten Ruine kaum zu begründen. Ich argumentiere zur Erklärung ikonologisch aus den bildhaften Qualitäten des Objektes selbst und ikonografisch aus den Deutungsmustern, die sich aus seiner Repräsentation im abbildenden Medium der Fotografie ableiten und durch die Bildmetapher der Kathedrale stützen lassen.

Eine Anmerkung zur Methode: Da es sich bei dem Untersuchungsgegenstand um eine Fotografie und somit technisch um ein Spurbild (Doelker 1997, S. 70), hinsichtlich des Verhältnisses von Vorlage und technischem Reflex um ein Abbild, um Mimesis handelt, denotiert das Bild etwas, dessen ikonische Extension für die Common-SenseBetrachtung von massenmedialen Bildern unproblematisch ist. Mithin können komplexe philosophische Fragen der Repräsentation für das hier verfolgte bildsoziologische und rezeptionsästhetische Erkenntnisinteresse außer Acht gelassen werden (z. B. stellvertretend Sachs-Hombach 2001). Sie sind nicht obsolet; die Beweisführung kann sie hier aber hintan stellen und gleichwohl Plausibilität reklamieren.

\section{Der Argumenttyp der metaphorischen Bildargumentation}

In der spezifischen fotografischen Inszenierung durch Frank Schwere löst die Ruine Assoziationen aus. Diese begründen sich extensional, in ihrer sichtbaren Erscheinung. Sie akzentuieren jeweils besondere Aspekte des abgebildeten Objektes einerseits, des Figur-Grund-Arrangements andererseits. Architektonisch ähnelt das Trümmerstück der gotischen Kathedrale. Die vertikalen parallelen Rippen der äußeren Tragekonstruktion rücken das Strebewerk der Hochgotik vor Augen, wie es sich etwa in der Kathedrale von Reims vollendet darstellt.

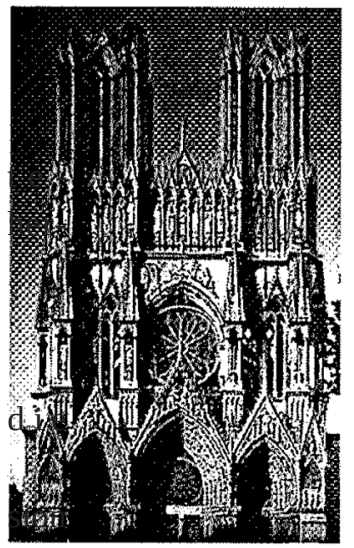

Gereihte spitzbogige Portale und der aus der Zerstörung entstandene „Stufengiebel“ unterstützen diese Lesart. Sie wird pointiert durch die Adorationsperspektive, den aufwärts gerichten Blick, der das Objekt erhaben erscheinen lässt und aus dem aus wir realiter die himmelan strebenden Kathedralen erblicken würden.

Zwar gibt es - mindestens - ein konterkarierendes Deutungsmuster: die Lesart als Turm von Babel. Hierzu würde sich Form der Spitze fügen, ebenso die rippenartige Struktur der Oberfläche - dies allerdings nur in der weniger bekannten Vervon Pieter Bruegel dem Älteren (Turmbau zu Babel, um 
1560). Die Semantik des Turms von Babel wäre, übertragen auf das World-Trade-Center, allerdings kontraproduktiv, wenn man sie in das vom SPIEGEL durch die Legende: „New York. Die verwundete Stadt“ akzentuierte Deutungsmuster einlesen wollte. Denn der babylonische Turm wurde von Gott zerstört; seine Auslöschung ist in christlicher Deutung die gerechte Strafe für menschliche Hybris. Die bildnerischen Mittel pointieren hier indessen, wie auch vielfach sonst, eines von mehreren möglichen Deutungsmustem. Die Wahl der Tageszeit, die Lichtsituation, die optische Isolierung durch Elimination ablenkender Hintergrundgebäude: All dies apostrophiert die Anmutung einer Bedeutsamkeit, die über die bloße Präsenz des reinen Erscheinens hinausweist. Die Restfassade steht, leicht gebeugt, aber immer noch eindrucksvoll, mittig in einer bergenden Schale aus Dunst, durchstrahlt vom Gegenlicht der dahinter aufgehenden Sonne. Solche Diaphanie hat ihre ikonografische Tradition in der Romantik, von vielen Beispielen seien nur die „Gotische Kirche auf einem Felsen am Meer bei Sonnenaufgang“ (1815) von Karl Friedrich Schinkel (Berlin, Alte Nationalgalerie) genannt, eine fiktive gotische Sakralarchitektur, mächtig auf dem Felsen gelegen, die Einbuchtung eines Meeresarmes überragend; ebenso die in das Diagramm aufgenommene „Kathedrale“ von Caspar David Friedrich (um 1818), die dem Figur-Grund-Bezug bei Frank Schwere sehr nahe kommt.

In der wechselseitigen Bestätigung ihrer Konnotationen legen Figur und Grund die bildmetaphorische Übertragung zwischen der Ruine des World Trade Center und der gotischen Kathedrale nahe. Es ist dabei natürlich dem Einwand zu begegnen, solche

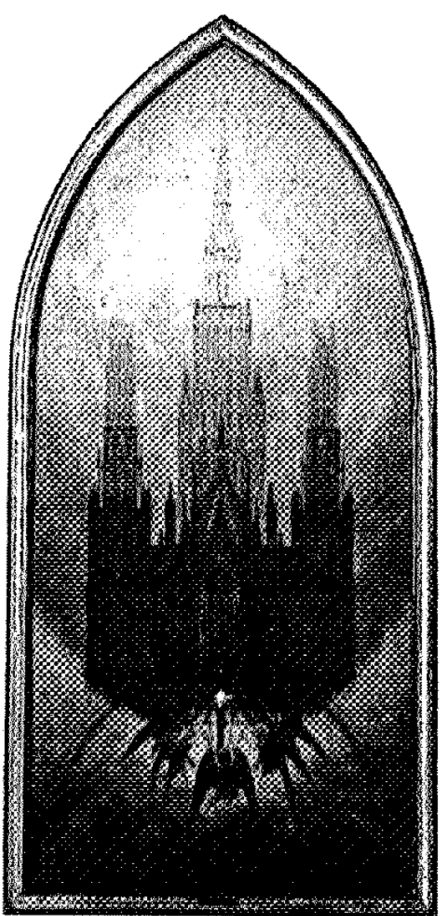
Assoziationen stellten bildungsbürgerliche Partikularkenntnisse dar; sie seien dem kollektiven Bildergedächtnis fremd. Dem lässt sich entgegenhalten, dass ehemalige Hochkulturschemata durch trickle-downEffekte längst in Populärkulturen durchgesickert und weithin verbreitet sind. Wenn wir nicht von der Gotik, sondern vom Gotisierenden sprechen, so ist an Gothic als durchaus metaphysisch inspirierte Jugendkultur zu denken (z.B. Hitzler 2001, S. 69-81), an Comics, Zeichentrick- und Fantasyfilme (Batman); das Gegenlicht der aufgehenden Sonne transportiert seinen Gestus der Verheißung und Rettung unter vielem anderen durch zahllose Hollywoodfilme. Es ist ja hier von genaueren Bezügen zum Zwecke einer Analyse die Rede - für die emotional wirksame und mehr geahnte denn durchschaute Wirkung genügt ganz im Sinne von Roland Barthes der mythische Hof des Begriffes als eine „nebulöse Kondensation“ (Barthes 4/ 1976, S. 99). 
Argumentationsanalytisch handelt es sich bei der metaphorisch gestützten Bildargumentation um den Typ des induktiven Analogieschlusses. „Er gründet sich auf einen Vergleich zwischen Dingen zweier verschiedener Arten. Man weiß, dass die Dinge der einen Art den Dingen der anderen Art in gewisser Hinsicht gleichen. Weiter weiß man, dass die Dinge der ersten Art eine bestimmte Eigenschaft besitzen; man weiß aber nicht, ob auch die Dinge der zweiten Art diese Eigenschaft besitzen. Der Analogieschluß besteht nun darin, dass man aus der Tatsache, dass sich die Dinge der der zwei verschiedenen Arten in bestimmter Hinsicht gleichen, schließt, dass sie sich auch in anderer Hinsicht gleichen“ (Salmon 2001, S. 197). Für die Bildmetapher ist es hilfreich, Extension und Intension der Bildbedeutung zu unterscheiden. Extensional, das heißt, im Hinblick auf ihre sichtbare Gestalt, ähnelt die Ruine des World Trade Center der gotischen Kathedrale. Diese ist steingewordene Bedeutung. In ihr inkarniert sich das eschatologische Programm von der Schöpfung über das christliche Heilsgeschehen bis zum Jüngsten Gericht. Die Westfassaden etwa erzählen dies (auch dem Analphabeten) wie ein offenes Buch (z.B. Jantzen 1987; Sedlmayr 2001). Zwar mag der Glaube durch Säkularisierung vielfach verloren sein, doch repräsentiert die Kathedrale immer noch eine christliche Wertegemeinschaft. Solche als kulturprägend verstandenen ethisch-moralischen Grundüberzeugungen (siehe die derzeitige Einwanderungsdebatte) sind der Kathedrale semantisch eingeschrieben, auf ihren Abbildungen stellen sie die intensionale Dimension ihrer Bedeutung dar. Intensional wird nun suggeriert, dass auch die Bedeutung der Ruine des World Trade Center der Bedeutung der gotischen Kathedrale entspricht. Die Evidenz der sichtbaren, also extensionalen Ähnlichkeit stellt sozusagen die Brückenköpfe bereit, auf denen sich die intensionale Bedeutungsbrücke auflagern kann. Dabei hat schon die extensionale Ähnlichkeit den Charakter einer Proposition, also des Kerns jeder Argumentation: Von Konzept A wird Konzept B ausgesagt:

Extensionale Proposition:

\begin{tabular}{|c|c|c|}
\hline Konzept A & Prädikation & Konzept B \\
\hline \multicolumn{3}{|l|}{ „Die Ruine des } \\
\hline World Trade Centers & sieht aus wie & eine gotische Kathedrale“ \\
\hline Referenz & & Referenz \\
\hline
\end{tabular}

Auf der intensionalen Ebene stellt sich der Analogieschluss her:

\begin{tabular}{|ll|}
\hline Konzept A & Konzept B \\
bedeutet & bedeutet \\
Konzept A'; & Konzept B` \\
wird als Empfängerfeld & (,eine gotische Kathedrale (B) \\
einer Metapher durch & bedeutet \\
& das christliche Abendland als Wertegemein- \\
Konzept B‘ & schaft \\
überlagert & $\left.\left(\mathrm{B}^{\prime}\right)^{`(}\right)$ \\
\hline
\end{tabular}


Konzept A verdoppelt sich zunächst als Bedeutung seiner eigenen Sichtbarkeit, als Referenz. Metaphorisch gelesen wird es auf Grund der extensionalen Analogie aus Konzept B mit suggeriertem Sinn gefüllt:

Vgl. Diagramm „Die bildsprachliche Proposition. Referenz und Prädikation“

Intensionale Proposition

\begin{tabular}{|c|c|c|}
\hline \multirow[t]{2}{*}{ Konzept A } & \multirow[b]{2}{*}{ Prädiktion } & \multirow[t]{2}{*}{ Konzept B } \\
\hline & & \\
\hline „Die Ruine des World Trade & & $\begin{array}{l}\text { „Eine gotische Kathedrale } \\
\text { (B) }\end{array}$ \\
\hline Centers (A) bedeutet auf Grund ihres & Wenn Konzept A aussieht & bedeutet das christliche \\
\hline $\begin{array}{l}\text { Aussehens das christliche Abendland } \\
\text { als Wertegemeinschaft (B’)“ }\end{array}$ & $\begin{array}{l}\text { wie Konzept B bedeutet es } \\
\text { das Gleiche wie Konzept B }\end{array}$ & $\begin{array}{l}\text { Abendland als Wertege- } \\
\text { meinschaft (B')“ }\end{array}$ \\
\hline
\end{tabular}

Die signifikanten Hinsichten der Analogie sind in diesem Falle die gereihten Spitzbögen, das filigrane Strebewerk, der Stufengiebel, die Diaphanie, welche durch die Fassade ohne Innenleben sogar noch gesteigert ist.

Als zutreffend angenommen wird hier als Unterstützung der Prädikation die Prämisse der sprechenden Architektur. So fasst z.B. Umberto Eco Architektur als semiotisches System auf:

„Ein Stuhl sagt mir vor allem, dass ich mich draufsetzen kann. Aber wenn der Stuhl ein Thron ist, dient er mir nicht nur zum Sitzen; er ist dazu da, sich mit einer gewissen Würde auf ihn zu setzen und bekräftigt den Akt des ,mit Würde Sitzens' mittels einer Reihe von Nebenzeichen, die Majestät konnotieren (Adler auf den Armlehnen, hohe Rückenlehne mit einer Krone oben auf etc.). Diese Konnotationen „majestätischer Würde“ sind in dem Maße funktionell, dass - wenn überhaupt vorhanden - man die Funktion das „Bequem Sitzens` vernachlässigen kann“ (Eco 1994, S. 311). Architektur aller Kulturen denotiert funktionszuweisende (Wohnhäuser, Bürohäuser, Schulen etc.) und konnotiert bedeutungszuweisende Zeichen (abweisende Herrschaftsarchitektur, demokratischtransparente Architektur etc.), diese sind in ihrer Spezifik allerdings kulturell verankert. Bildförmige Argumente funktionieren innerhalb von kulturspezifischen Weltbildern und Bilderwelten; sie beruhen auf den symbolischen Ordnungen von Kulturen und verankern diese im kollektiven Bilderhaushalt ihrer Mitglieder.

\section{Spezifika nachhaltiger Argumentation: Schlussregel, Einschränkungsbedin- gungen und Folgediskurse}

Die metaphorische Interpretation stellt sich als ein Evidenzerleben ein: Mir als Betrachter fällt ein bildhafter Bezug auf, in diesem Fall eine Ähnlichkeit 
auf Grund bestimmter Details. Ich verknüpfe das Bild mit anderen Fundstücken meines imaginären Museums und leite daraus eine Rezeptionsmöglichkeit ab. Mir fällt z.B. durch einen emergenten Vergleich „das Gotische“ auf. Ich frage, „warum“ und welche Bestandteile der Ruine und ihres Hintergrundes den gotisierenden Eindruck akzentuieren. Die Bestandteile erfüllen die argumentative Funktionsstelle der Schluss-rege/, der Begründung für den Weg vom Datum zur Konklusio (entsprechend etwa dem verbreiteten Schema von Stephen Toulmin 1996, S. 88-98). Die Zeichnung von Viollet le Duc (19. Jh.) soll in dem Schaubild die visuelle Schlussregel illustrieren: Hier ist das Gotische in extenso ausformuliert - eine siebentürmige Kathedrale, die indessen so nie gebaut wurde.

Allerdings wirkt die Ruine des World Trade Center nur gotisierend, ohne doch gotisch zu sein - es treten Ausnahmebedingungen (rebuttal) hinzu - etwa, dass das Trümmerstück nur einen Turm hat, nur Fassade ist usw. Solche Ausnahmebedingungen schränken die Gültigkeit der Konklusio ein. In der bildimmanenten Argumentation erfüllen sie die Funktion von Gelenkstellen, die Folgediskurse in Gang setzen (siehe unten).

Solange die Analyse auf der syntaktisch beschreibbaren Ebene des sichtbaren Befundes, der bildnerischen Mittel, der innerbildlichen Bezüge, der Bildbestandteile verbleibt, bewegt sie sich auf der Ebene der Extension bzw. der Referenz eines Abbildenden auf außerbildlich material faktisch oder mental als Entwurf Vorfindliches. Wenn man zur Ebene der jeweiligen Bedeutungen wechselt, so geht man vom gezeigten Gegenstand zum gemeinten Sinn über, von der Extension zur Intension. Dieses setzt kulturell spezifische Kenntnisse voraus, im Sinne Erwin Panofskys ikonologisches Wissen, allerdings, wie oben gezeigt, auf verschiedenen Ebenen der Ableitung. Die visuelle Argumentation lässt sich dann auf dieser Inhaltsebene wie folgt spezifizieren: Ich interpretiere nun die Zerstörung eines Bauwerkes symbolisch, als Angriff auf das, wofür es steht, in der bildrhetorischen Figur des pars pro toto (als Teil für das Ganze). D.h. ich vollziehe in der Schlussregel eine Deduktion. 


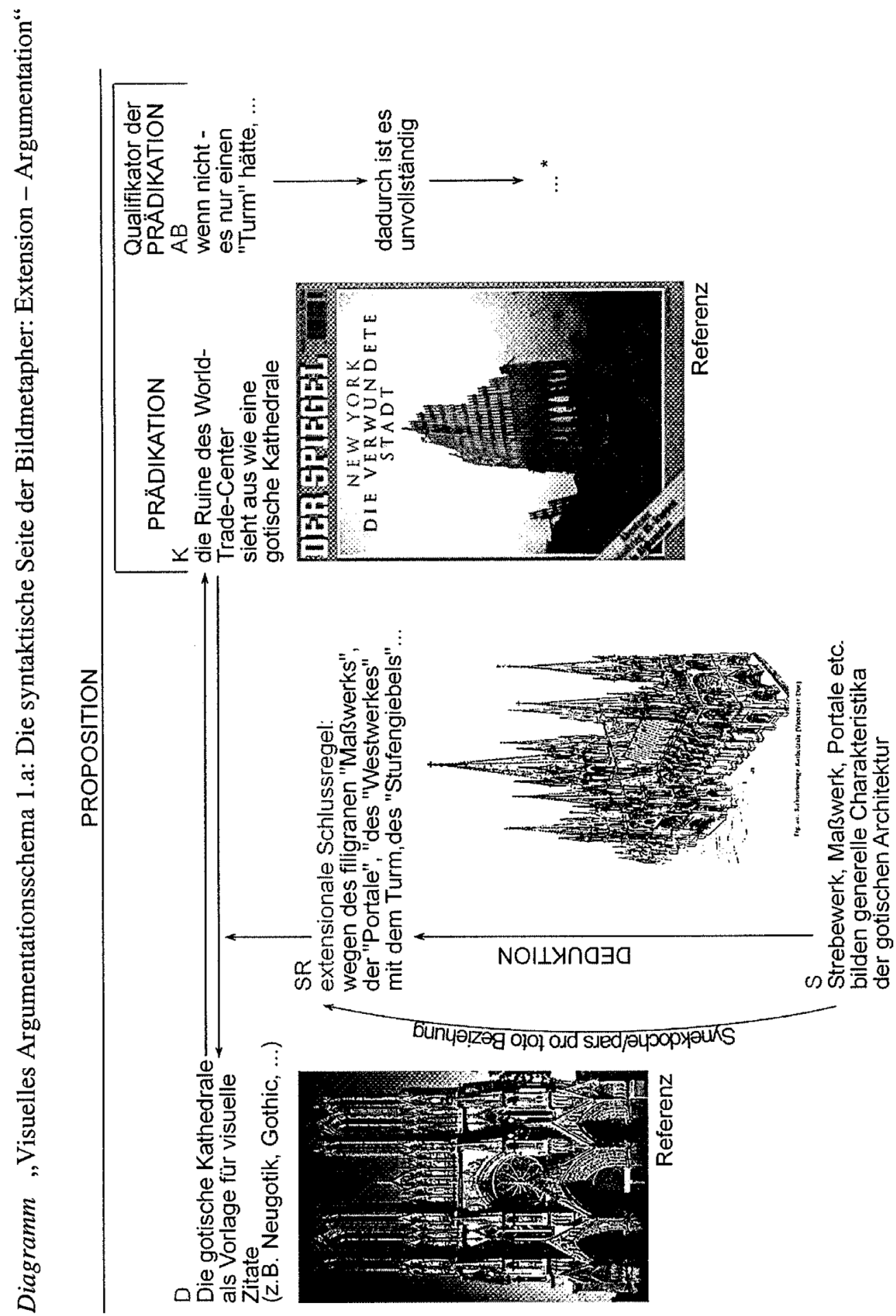




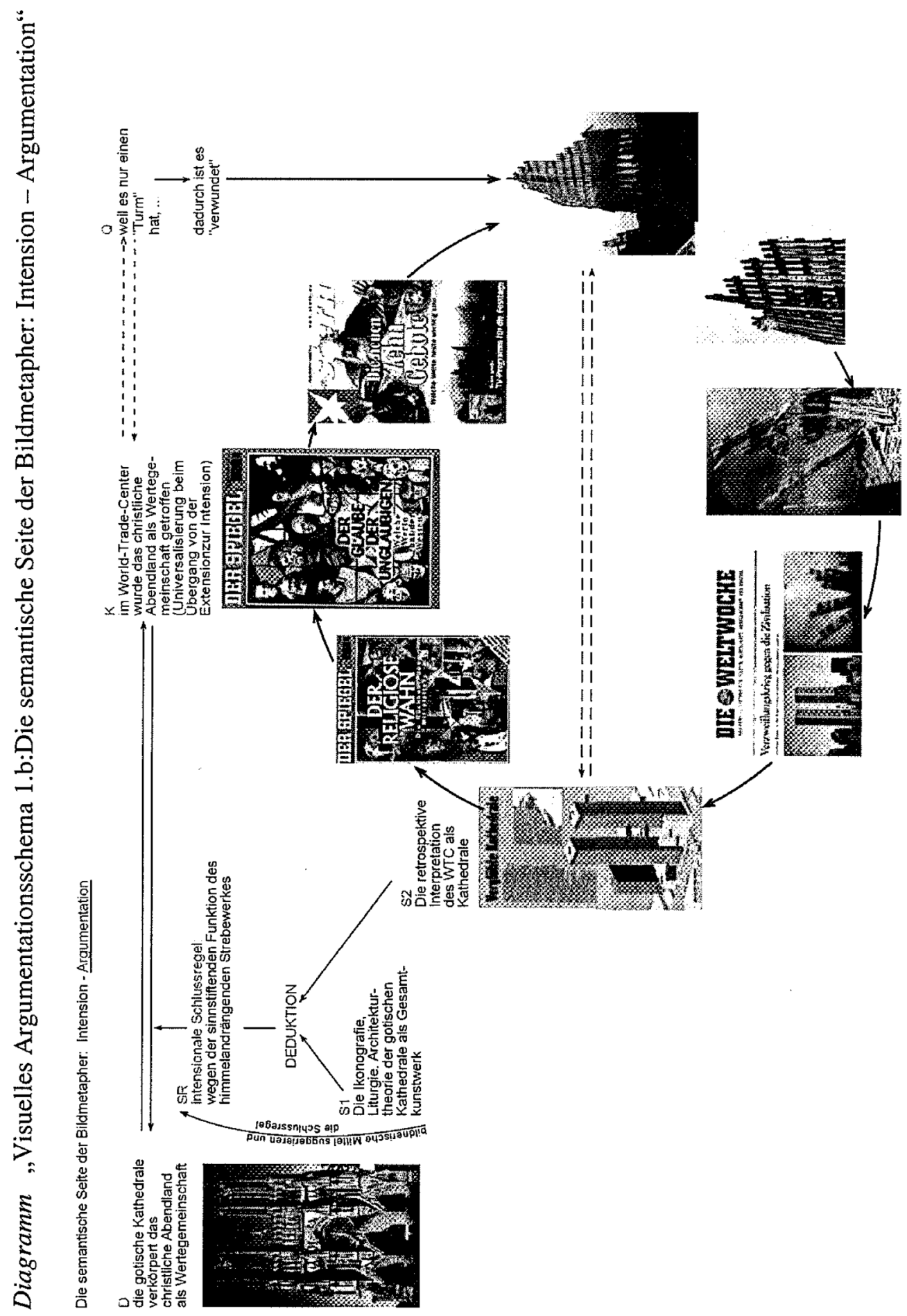


Dabei behalte ich die extensional erschlossene gotisierende Analogie bei und leite den Bildsinn (wohlgemerkt den Bildsinn und nicht die mögliche Intention des außerbildlichen Terroranschlages) aus dieser bildrhetorisch inszenierten Analogie ab. Es wurde dann das christliche Abendland als Wertegemeinschaft getroffen. Einmal auf diese christlich affizierte Schiene gebracht, kann sich nun der interpretative Diskurs auf diesen Gleisen weiterbewegen und potenzieren. Es ist sozusagen ein Rad oder besser eine Spirale angestoßen, die sich nun dreht. Die Bilder in diesem gleichen Bezugsfeld eines christlich affizierten Wertediskurses stützen sich gegenseitig - umso mehr es werden, desto fester wird die Stützung, das backing, desto selbstverständlicher die Quer-verweise, desto abwegiger erscheinen generelle Infragestellungen. Der Diskurs wird selbstreferentiell, quasi autopoietisch. Die Spirale des Schaubildes zeigt einige Visualisierungen der Folgediskurse, die die unterstellte Rückkehr des Religiösen durchbuchstabieren: Die eschatologische Etikettierung der Ruine als Apokalypse („Apokalypse now“, eine Anspielung auf Coppolas Film, der aber seinerseits schon den Vietnamkrieg metaphysisch hypostasiert); die implizite Grenzziehung zwischen Zivilisation und Barbarei als „Verzweiflungskrieg gegen die Zivilisation" in der WELTWOCHE; die retrospektive Deutung des WTC als „Verglühte Kathedrale“ (nach dem Anschlag);,,Der religiöse Wahn“ und „Der Glaube der Ungläubigen“ (SPIEGEL), Artikel, die eine ethischmoralische Selbstvergewisserung und Grenzziehung artikulieren und der Weihnachtsausgabe des stern (52/19.12. 2001) „Die neuen Zehn Gebote“ als konsensfähigem (oder so unterstelltem) Kanon den Weg bereiten. Alle arbeiten mit der Evidenz des Bildhaften: Gezeigtes ist als Position zunächst einmal der Fall („Ein Bild kann nicht ,nein“ sagen“); Ein Bild wie das des stern, das einen Assoziationshof von Schutz und Segen in einer Kombination aus Fiktivem (Gottvater) und Abgebildetem (die neue Skyline New Yorks vor der Morgenröte) assoziiert, immunisiert sich gegen Kritik weitaus stärker, als es eine plakative Schlagzeile, etwa „Das Religiöse kehrt zurück“ je zu leisten vermöchte.

\section{Orientierungsmetaphern im zeitlichen Verlauf}

Auf dem Zeitstrahl schiebt sich das Verweisungsfeld des Hier und Jetzt meines Sprechens und Zeigens, aber auch meiner Wahrnehmung von Gezeigtem, also von Bildern, „aufwärts“, zwischen dem Rückblick auf die Vergangenheit und dem Ausblick auf die Zukunft. Die Bildmetapher muss dabei notwendigerweise immer zurückgreifen - das Spenderfeld der Metapher muss älter sein als das Empfängerfeld, es muss ihm zeitlich vorausliegen. Etwas kulturell Bekanntes (hier die gotische Kathedrale), ein Bestandteil des imaginären Museums der Betrachter, wird in der visuellen Metapher auf etwas Neues (hier die Ruine des WTC) angewandt und interpretiert das Neue um, lässt es in einem besonderen Lichte erscheinen. Insofern trägt die visuelle Metapher den bildhistorisch rückwärtsgewandten Teil zur visuellen Argumentation bei. Lakoff/Johnson nennen dies ,Strukturmetaphern', „Fälle, in denen ein Konzept von einem anderen Konzept her metaphorisch strukturiert wird“ (LakoffrJohnson 2000, S. 22). 
Diagramm „Visuelles Argumentationsschema (2): Die semantische Seite der Bildmetapher: Intension - Metaphorisierung

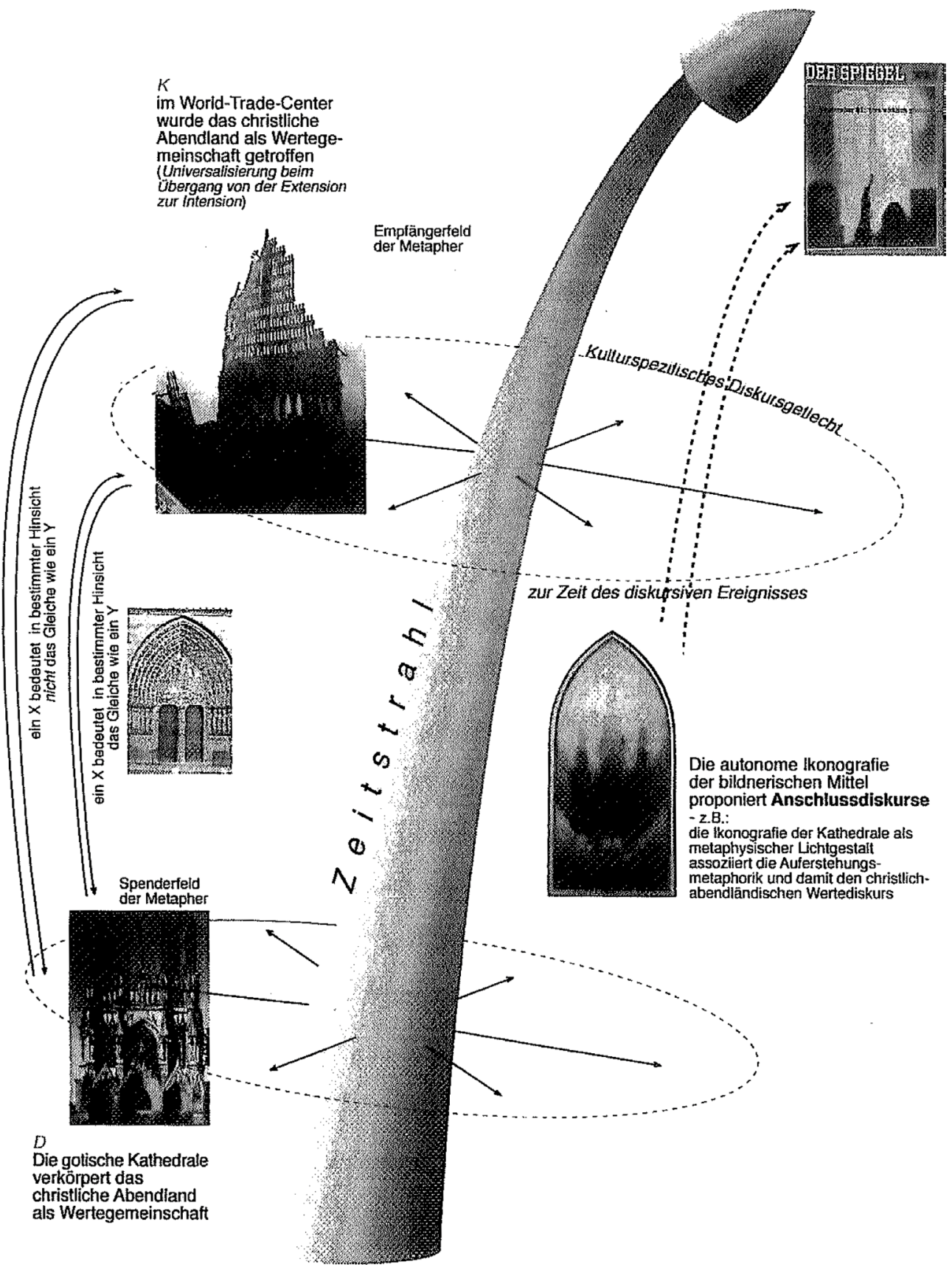


Die Brücke zwischen dem Neuen und dem Bekannten wird durch gleiche Bestandteile beider und eine besondere Sichtweise, einen spezifischen point of view artikuliert, der eben dieses Gleiche als das tertium comparationis der Metapher hervorhebt. Insofern begründet sich die visuelle Metapher aus der Prädikation „ein X sieht in einer bestimmten Hinsicht aus wie ein Y“. Das Gleiche wird durch besondere bildnerische Mittel akzentuiert, welche die metaphorische Übertragung suggerieren. In unserem Beispiel zählt zu den bildnerischen Mitteln etwa die Froschperspektive respektive Adorationsperspektive (Bewunderungsperspektive), aus der wir gewohnt sind, gotische Kathedralen realiter oder auf Bildern anzuschauen - eben zu ihnen „aufzublicken“. Metaphorisch, also nicht etwa identifizierend, ist der Übertragungsprozess aber nur, wenn er zugleich durch einen widerständigen Teil konterkariert wird: „Dieses $\mathrm{X}$ sieht in einer bestimmten anderen Hinsicht auch nicht aus wie ein Y.“

Eine Bildmetapher, die sich im Verstehensprozess des Betrachters als solche realisiert, kann in ihrer Semantik flankiert werden durch Bedeutungen, die ebenfalls historisch mit dem Spenderfeld der Metapher verbunden sind. Sie können ihre Zusatzbedeutung auf die Grundbedeutung der Bildmetapher aufsatteln, in unserem Fall etwa die Ikonografie der gotischen Kathedrale als metaphysische Lichtgestalt, in der die filigrane Transparenz des Strebewerkes von überirdischem Gegenlicht durchstrahlt, diaphan wird. Zum sakralisierenden Topos dieser Lichtgestalt gehört die Schalenform in der romantischen Malerei - die Morgenröte des neuen Tages bricht aus der bergenden Nebelschale hervor. In diesem Gefäß erscheint das gotische Bauwerk als ebenso geborgen und dem Licht entgegen gehoben, wie in dem geschlossenen Sinnhorizont seines Weltbildes. Dieses Weltbild assoziiert die Auferstehungsmetaphorik und damit den christlichabendländischen Wertediskurs. Die Einbettung einer Figur in einen kulturellen Grund leisten „Orientierungsmetaphern“. Durch sie wird „ein ganzes System von Konzepten in ihrer wechselseitigen Bezogenheit organisiert.“ Solche Metaphern sind kulturspezifisch und leiten sich vielfach aus räumlicher Orientierung ab. „Beispielsweise liegt in einigen Kulturen die Zukunft vor den Menschen, während sie in anderen Kulturen hinter den Menschen liegt“ (Lakoff/Johnson a.a.O., S. 22). Für die abendländische Kultur ist prägend, dass das Zukünftige kompositorisch oben, das Vergangene unten angeordnet wird. In diesem Sinne hat die verbale und visuelle Metapher eine Verankerungsfunktion in unseren konzeptuellen Deutungssystemen: Sie macht das Neue mit den vertrauten Interpretationen kompatibel. Zugleich legt sie nahe, auf das „Unerhörte“ mit den vertrauten Mechanismen zu reagieren. Metaphorisierung gibt die verlorene Handlungskompetenz zurück. Ein Zitat von Lakoff/Johnson lässt sich passgenau auf den bildhaften Umgang mit dem 11. September beziehen: „Metaphern können für uns Realitäten schaffen, vor allem soziale Realitäten. Auf diese Weise kann eine Metapher Orientierung geben für unser zukünftiges Handeln. Solchermaßen geleitetes Handeln passt natürlich zu der entsprechenden Metapher. Dadurch wird wiederum die Metapher in ihrer Fähig- 
keit gestärkt, unsere Erfahrungen kohärent zu machen. In dieser Hinsicht können Metaphern sich selbst erfüllende Prophezeiungen sein“ (Lakoff/Johnson a.a.O., S. 179). So macht z.B. die Metaphorisierung „Wunde“ (SPIEGEL Nr. 43/01) die Anschlussmetapher „Heilung“ möglich und schlüssig, und in der Visualisierung des 2. Golfkriegs als chirurgisch präzise „Operation“ (im doppelten Sinne) stand bereits die handlungsanleitende Folgemetapher auf eine Verletzung „Gewehr bei Fuß“. Das metaphorische Konzeptsystem „christliches Abendland als Wertegemeinschaft“, präformiert als Basis die Deutungsmuster, es geniert, wie gezeigt, Rückkopplungsschleifen. Darin erscheint es nur folgerichtig, wenn auch das retrospektiv zur Kathedrale umgedeutete World Trade Center selbst metaphysisch, z.B. als Lichtprojektion, wieder aufersteht (SPIEGEL-Titelbild Nr. 49 vom 3.12.01).

\section{Dialektische Antithetik}

Wenn im World Trade Center das „Abendland“ als Wertegemeinschaft getroffen wurde, wie es die Bildmetapher suggeriert - und wie es übrigens viele Kommentare auch verbal deuten -, dann bildet dies im dialektischen Verständnis der Argumentation die Ausgangsthese. Unterstellt wird den Attentätern, dass dieser symbolische Gehalt, der nun retrospektiv auf das Gebäude projiziert wird, auch gemeint war. „Ein Angriff auf das Herz der westlichen Welt“ (kultuRRevolution 43/2002), das WTC als Pumpe der Kapitalströme, des Blutes in den Lebensadern des Kapitalismus oder der Freien Marktwirtschaft, so und ähnlich wird variantenreich die Kollektivsymbolik des westlichen Interdiskurses evoziert.

Die Antithese wäre die, dass ein kohärentes Wertesystem der „Freien Welt“, das die Kraft besäße, kollektive Identität zu vermitteln, gar nicht mehr existiere, und dass gerade die Ruine - mit dem gleichen metaphorischen Bezug - Ausdruck seiner Zerrüttung oder zumindest wehrlosen Schwäche sei. Die dialektische Synthese aus beiden Lesarten wäre eine aus den Fugen geratene Welt, eine Verwirrung der globalen Werteordnung, ein Rückfall hinter Humanismus und Aufklärung, den der Spiegel in der ersten Deutungsphase als „Rückkehr des Mittelalters“ (SPIEGEL: „Der religiöse Wahn“, Nr. 41/01) qualifiziert. Es bliebe dann die kontingente Frage: Aus welchen Fugen ist diese Welt geraten und aus wessen Sicht? Durch solche Fragen könnte die dialektische Antithetik aus der Umklammerung durch die hegemonialen Diskurse (Jürgen Link) ausbrechen; verschiedene Nicht-Regierungsorganisationen (NGOs) verfolgen in diesem Sinne Strategien von Gegenbildern, etwa attac. 
Diagramm „Visuelles Argumentationsschema (3): Die semantische Seite der Bildmetapher: Intension - visuelle Dialektik"

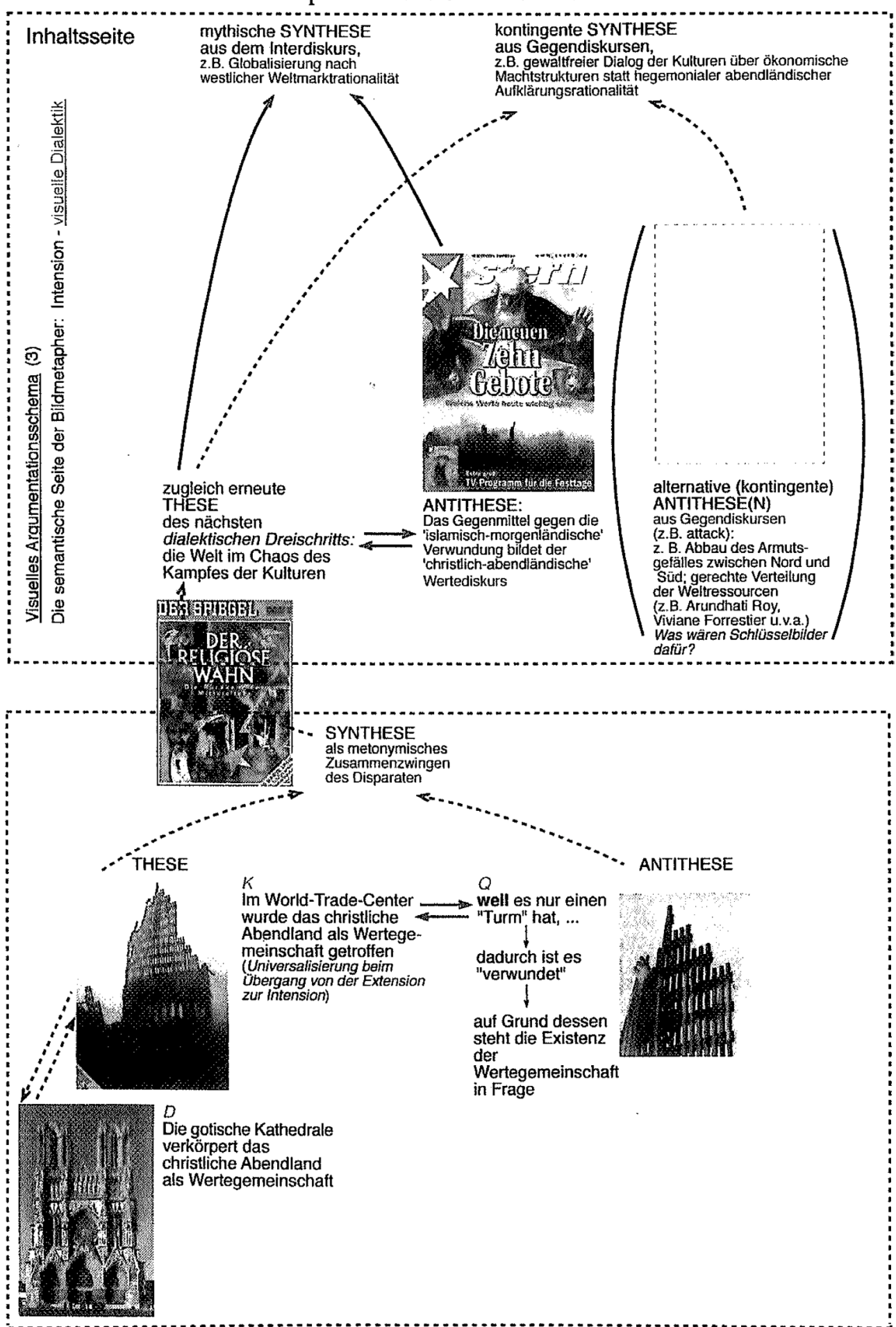


Anarchie und metaphysische Überhöhung waren die Deutungsmuster der ersten Stunde - natürlich können sie nicht das letzte Wort bleiben. Die imbefriedigende Synthese des Konflikts („Rückkehr des Mittelalters“, SPIEGEL Nr. 41/01) muss aus ihrer eigenen Unzulänglichkeit heraus eine neue Antithese gebären - die Neubesinnung auf den Wertekanon eines christlichen Abendlandes, allerdings in säkularisiertem Gewände: die Beibehaltung einer christlichen Ethik (Gottvater auf dem „stern“: „Die neuen zehn Gebote“ (stern Nr. 52/01); angereichert um naturwissenschaftlich-technische Erkenntnisse und um Toleranz („Welche Werte hat der Westen“ - die Forscherpersönlichkeiten und Philosophen auf dem SPIEGEL Nr. 52/01) sowie um Art. 20 (4) des Grundgesetzes: das Widerstandsrecht in der wehrhaften Demokratie. „Die ,wehrhafte Demokratie im Angriffskrieg. Von der Ausbildung eines neuen, einheitlichen Paradigmas der Sicherheitspolitik“ heißt dazu die Analyse in der Zeitschrift kultuRRevolution 43/2002. Bei der so konstruierten Frontstellung „Moderne gegen Mittelalter“ bleiben die Hintergründe der Weltwirtschaft ausgeblendet - nicht nur in den Visualisierungen, auch in den entsprechenden Texten. Die Bilder übernehmen die Funktion der Meinungsführerschaft - sie werden vor ihren zugehörigen Texten wahrgenommen und markieren deren Programm. Was in ihrem Deutungshorizont ausgeblendet bleibt, kommt auch in den entsprechenden Texten nicht zur Sprache. Der Interdiskurs verbleibt in der idealistischen Frontstellung des Ideellen, welche wie einen Abwehrschild Werte aufrichtet (Huntingtons „Kampf der Kulturen"), die Handlungsmotive der Gegenseite aber ignoriert und tabuisiert. So wird etwa in den Visualisierungen eine arabische Lesart neutralisiert, der Anschlag stelle eine Reaktion auf den Ausverkauf saudischen Erdöls durch eine unermesslich reiche Herrscherclique dar, auf die Stationierung ungläubiger Truppen im Land der heiligen Stätten, auf die Doppelzüngigkeit des ,Westens` im Umgang mit UN-Resolutionen, die Israel und den Irak betreffen usw. Im Falle des World Trade Center oszilliert der Interdiskurs um die immer neu ausstaffierte Grenze zwischen Zivilisation und Barbarei (Weltwoche: „Verzweiflungskrieg gegen die Zivilisation“); wobei die Wertungen natürlich immer zugunsten der eigenen Seite gewichtet sind.

Kritische Gegenpositionen (etwa Noam Chomsky, Arundhati Roy, Susan Sontag, Johan Galtung) visualisieren ihre Erklärungsmodelle nicht. Vielleicht würden sie mehr Öffentlichkeit erreichen, wenn sie sich der bewusstseinsprägenden Kraft der Bilder bedienten. Wenn etwa der Friedensforscher Johan Galtung sich dagegen verwahrt, den 11. September als eine „Stunde Null“ zu qualifizieren, vielmehr eine Reaktion auf die Erfahrung von kultureller und wirtschaftlicher Hegemonie sieht, so wäre dafür ein kraftvolles Schlüsselbild zu entwerfen und in der Diskussion zu positionieren. Kontradiktorische Deutungen etwa aus der arabischen Welt werden bei uns nicht thematisiert. Welche hiesige Zeitschrift übersetzt und druckt Kommentare arabischer Zeitungen? Unsere Bildwelten und Feindbilder sind hermetisch - ebenso wie ihre. Eine aufgeklärte Demokratie sollte den Disput aushalten, der den Gegner zu Wort und sozusagen „zum Bild“ kommen lässt. 


\section{Fazit: Bildsprachenkompetenz als Kern von Medienpädagogik}

Die hier vorgestellten Deutungsmuster für die Bildargumentation in Schlüsselbildern im Kontext visueller Diskurse setzen als Argumentation, Metaphorik und Dialektik unterschiedliche Untersuchungsschwerpunkte und ergänzen sich darin. Sie leisten einen Beitrag zur Begründung und Erklärung des Umstandes, dass Bildwahmehmung kulturspezifische Weltdeutung in aller Regel nicht in Frage stellt, sondern bestätigt. Durch die Mittel der analytischen Erhellung und des praktischen Konterkarierens durch Gegenbilder zeichnen sich aber Werkzeuge ab, die kritisch aufklärende Gegenbilder produzieren können.

Die impliziten Argumentationen in Bildern zu erkennen, bildet einen Bestandteil von Bildsprachenkompetenz, diese ihrerseits stellt, diesseits von bloßem Handling, ein inhaltliches Fundament für Medienkompetenz bereit. Schließt man sich Dieter Baacke an, der Medienkompetenz in einen noch umfassenderen Bereich von kommunikativer Kompetenz einlagert, so schließt sich der Kreis: „Genau dies meint Kompetenz: Es geht um mehr als um die Fähigkeit, eine neue Technik (etwa den online geschalteten Computer) handhaben zu können; verbunden ist diese technisch-praktische Fertigkeit mit dem Vermögen der Menschen, sich Gedanken über etwas zu machen, kritische Argumente zu formulieren, aber auch mit Hilfe von Lektüre Genussfähigkeit zu erlangen“ (http://www. paedagogik.uni-bielefeld.de/agn/ag9/MedKomp.htm). Das Formulieren von Argumenten setzt das Erkennen von Argumenten voraus; für eine Mediengeneration insbesondere im Medium des Bildhaften, in dem sie sich alltagspraktisch in Gestalt von Jugendszenen auch artikuliert. Wenn gerade deutsche Schülerinnen und Schüler zu $42 \%$ angeben, sie würden „nicht zum Vergnügen lesen“ (Nur in Belgien, den Niederlanden und Japan liegt dieser Anteil höher (PISA 2000, S. 114)), so sollte als eine Konsequenz die passiv vorhandene Bildlesekompetenz in die aktive Verfügung transferiert und für verbalsprachliche Kompetenz fruchtbar gemacht werden. Bildsprachliche Kompetenz ist nicht in dem Maße wie Verbalsprache an restringierten oder elaborierten Code gebunden und kann daher zum Abbau sozialer Defizite im Beherrschen der Wortsprache beitragen. Mit dem Fokus auf Inhalte kann Medienpädagogik der technizistischen Verkürzung des Kompetenzbegriffs entgehen, wie sie etwa Hans-Dieter Kübler kritisiert: „So stehen im Vordergrund das Handling der Computer- und Internettechnologie (obwohl diese voraussichtlich in einigen Jahren gänzlich anders aussehen wird) und die Beherrschung der marktgängigen Software (...). Die kommunikative Durchdringung oder Hinterfragung der klassischen Medien(strukturen), worüber sich früher Medienkompetenz definierte, ist kaum mehr gefragt oder in medienpädagogische Nischen verschoben“ (Kübler 2002, S. 6).

Die PISA-Studie benennt drei kulturelle Basiskompetenzen, die sie zu „kultureller Literalität“ (PISA 2000, S. 20f.) integriert: die muttersprachliche, mathematische und naturwissenschaftliche Kompetenz. Zu wenig beachtet 
wird der Umstand, dass Sprachen und Kommunikation einem ständigen Wandel unterliegen, der beim Messen von Kompetenzen mit einbezogen werden sollte.

Ohne den Kompetenzbegriff inflationieren zu wollen, sollte doch vor dem Hintergrund der „Visuellen Zeitenwende“ der Stellenwert einer visuellen Basiskompetenz als ein „basales Kulturwerkzeug“ (ein Begriff aus PISA) untersucht werden. Gerade dieser inhaltliche Beitrag der Medienpädagogik zum Wahrnehmungs- und Urteilsvermögen verschränkt sie mit den traditionellen Fächern des schulischen Kanons und verspricht eine Verbesserung im Bereich der verbalen Lesekompetenz. Fächerverbindendes Arbeiten etwa zwischen Kunst und Deutsch bietet sich hier an, wobei die Qualifikationen der Fachlehrer/-innen im Sinne der heute so beliebten „Synergieeffekte“ genutzt werden können. Dabei wird der Deutschlehrer durch sein sprachwissenschaftliches Teilstudium eine Terminologie und Systematik einbringen können, die der Kunstlehrer als „Augenmensch“ durch seine Sensibilität für die Zwischentöne von Bildern, für ihre Genauigkeit in der Errettung des Individuellen, auszugleichen vermag.

Im angelsächsischen Bereich hat sich „Visual Literacy“ als Begriff und Programm für eine Basisqualifikation etabliert; im deutschen ist ein Konzept „Bildsprachenkompetenz“ bislang kaum ausgearbeitet. Hier besteht Nachholbedarf. Wenn Diskursanalysen wie bisher auf verbale Kommunikation beschränkt bleiben, begibt sich der interkulturelle Dialog aussichtsreicher Chancen. Die Möglichkeiten eines Kulturvergleichs der Bilder auszuloten und in seinen Chancen abzuschätzen, bleibt Aufgabe weiterer Untersuchungen.

\section{Literatur}

Anderson, J.R.: Kognitive Psychologie. Heidelberg Berlin 2001.

Barthes, R.: Mythen des Alltags. Frankfurt/Main 1976.

Bayer, Kl.: Argument und Argumentation. Logische Grundlagen der Argumentationsanalyse. Opladen/Wiesbaden 1999.

Brekle, H.E.: Semantik. Eine Einführung in die sprachwissenschaftliche Bedeutungslehre. München 1972.

Chronik aktuell. Der 11. September 2001. Gütersloh/München 2001.

Doelker, Ch.: Ein Bild ist mehr als ein Bild. Visuelle Kompetenz in der Multimedia-Gesellschaft. Stuttgart 1997.

Eco, U.: Einführung in die Semiotik. München 1994.

Habermas, J.: Theorie des kommunikativen Handelns. Band 1: Handlungsrationalität und gesellschaftliche Rationalisierung. Frankfurt/Main 1985.

Hitzler, R./Bucher, Th ./Niederbacher, A.: Leben in Szenen. Formen jugendlicher Vergemeinschaftung heute. Opladen 2001.

Jantzen, H.: Kunst der Gotik. Klassische Kathedralen Frankreichs Chartres, Reims, Amiens. Berlin 1987.

Kutter, M.: Nachwort. In: Bilder im Kopf oder die Magie des Gedruckten. Pictures in our Minds or the Magic of Print. STERN Anzeigenabteilung. Hamburg 1987. 
Lakoff, G./Johnson, M.: Leben in Metaphern. Konstruktion und Gebrauch von Sprachbildern. Heidelberg 2000.

Ludes, P.: Schlüsselbild-Gewohnheiten. Visuelle Habitualisierungen und visuelle Koordinationen. In: Knieper, Th/Müller, M.G. (Hrsg.): Kommunikation visuell. Das Bild als Forschungsgegenstand - Grundlagen und Perspektiven. Köln 2001, S. 64-78.

Rötzer, F.: Das terroristische Wettrüsten. Anmerkungen zur Ästhetik des Aufmerksamkeitsterrors. In: Kunstforum 157/2001, S. 45-49.

Sachs-Hombach, K.: Bild und Prädikation. In: Sächs-Hombach, K. (Hrsg.): Bildhandeln. Interdisziplinäre Forschungen zur Pragmatik bildhafter Darstellungsformen. Magdeburg 2001, S. 55-76.

Salmon, W.C.: Logik. Stuttgart 2001.

Sedlmayr, H.: Die Entstehung der Kathedrale. Baukunst Mystik. Symbolik. Wiesbaden 2001.

Toulmin, St.: Der Gebrauch von Argumenten. Weinheim 1996. 